\title{
Consulta de cessação tabágica no Centro de Saúde de Alvalade Os primeiros 184 pacientes fumadores. Avaliação de resultados
}

\author{
LUÍS REBELO*
}

\section{RESUMO}

Introduç̃o: A cessação tabágica é um dos componentes mais relevantes para obter, num País, a baixa de prevalência dos fumadores a curto prazo. A descrição de uma consulta de cessação tabágica e a avaliação dos seus resultados pode ser útil.

Objectivos: Caracterizar a consulta de cessação tabágica do Centro de Saúde de Alvalade e avaliar a taxa de abstinência entre pacientes fumadores.

Metodologia: Estudo descritivo e transversal. A colheita de dados teve por base os elementos das fichas clinicas. Foram estudados 184 pacientes fumadores, correspondendo a fodos os que tinham completado à data, pelo menos, um ano após a primeira consulta. Utilizou-se o teste de Fagerström para avaliar a dependência física da nicotina e o teste de Richmond para determinar a motivação para deixar de fumar.

Resultados: Os fumadores estudados distribuem-se igualmente pelo sexo masculino e sexo feminino, têm uma idade média de 45 anos, exercem uma profissão e possuem o nível secundário de escolaridade. A média da idade em que fumaram o primeiro cigarro foi de 15,6 anos e fumavam em média 28 cigarros/dia. As mulheres fumavam menos cigarros que os homens, 11 a 20 cigarros versus 30 ou mais cigarros $(p=0,018) .0$ nivel de dependência da nicotina médio apresentado pelos fumadores foi moderado (5,9 pontos). Os homens obtiveram um valor superior ao das mulheres, 6,4 versus 5,3 pontos ( $p=0,001)$. As mulheres fumavam "para combater 0 stresse", os homens "por prazer». Por comparação com os homens, as razões para fumar - "combater o stresse» $(p=0,002)$ e "para não engordar» $(p=0,002)$ são de tipo feminino. A taxa de abstinência, avaliada 12 meses após a data da primeira consulta, foi de $24 \%$. Os fumadores com patologia psiquiátrica tiveram maior difficuldade em deixar de fumar $(p=0,046)$.

Conclusões: Os fumadores estudados são de ambos os sexos, têm uma média de idades de 45 anos, uma escolaridade média e começaram a fumar aos 15 anos. Fumam 28 cigarros por dia, apresentam uma dependência moderada da nicotina e estão motivados a deixar de fumar. Doze meses após a primeira consulta, $24 \%$ deles estava abstinente.

Palavras-Chave: Tabaco; Consulta; Cessação Tabágica.
*Médico de Família do Centro de Saúde de Alvalade (ARSLVT). Responsável pela Consulta de Tabagismo do Centro de Saúde de Alvalade. Professor da Faculdade de Medicina de Lisboa. Presidente da Confederação Portuguesa de Prevenção do Tabagismo

\section{INTRODUÇ̃̃O}

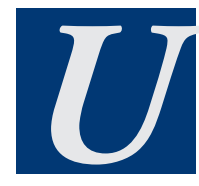

m país para reduzir o consumo de tabaco, e as suas consequências na saúde dos seus cidadãos, tem que ter uma política explícita e abrangente de prevenção e controlo do tabagismo.

Os estudos confirmam a necessidade de desenvolver várias abordagens, de diferente efectividade, mas sempre complementares. Está demonstrado que as abordagens mais importantes no controlo do consumo do tabaco são: uma política de preços elevados, a proibição da promoção e marketing dos produtos do tabaco, a restrição de fumar em locais públicos fechados, campanhas educacionais dirigidas aos fumadores e o apoio àqueles que decidam deixar de fumar mediante a disponibilização de consultas de cessação tabágica e de medicamentos.

Em 2001 o Centro de Saúde de Alvalade propôs um programa de "Promoção e Prevenção do Tabagismo» dirigido à população inscrita no centro de saúde e aos utentes das instituições comunitárias (incluindo as escolas) com as quais tem algum tipo de vínculo.

A consulta de cessação tabágica é um dos componentes desse programa. Teve início em Janeiro de 2002, com uma equipa constituída por dois médicos, uma enfermeira, uma psicóloga e uma nutricionista. Conta com o apoio administrativo do centro de saúde e o empenho do seu Director. As consultas 
realizam-se às $5^{\text {a }}$ Feiras, das 14 às $18 \mathrm{~h}$, em espaço próprio decorado e equipado para o efeito.

Foi elaborado um processo clínico específico com ficha de primeira consulta, ficha de seguimento e fichas de consulta de nutrição, de psicologia e de enfermagem. As fichas foram adaptadas do modelo existente no Hospital de Pulido Valente.

Foram instituídos e divulgados no centro de saúde e entre os médicos de família os seguintes critérios de acesso prioritário à consulta:

- Fumar o primeiro cigarro na primeira meia hora após acordar

- Estar motivado a deixar de fumar nos próximos 30 dias

- Fumar 20 ou mais cigarros por dia

- Não estar em crise psicossocial

- Ter as 5 $5^{\text {a }}$ Feiras à tarde livres e ser portador de carta do médico assistente.

A metodologia usada é a de "multi-componentes». ${ }^{2}$ Baseia-se num programa estruturado de consultas de apoio intensivo. O método é do tipo cognitivo-comportamental, compreende uma série de "entrevistas motivacionais" realizadas ao longo de um período de 3 a 6 meses, complementadas por uma prescrição farmacológica personalizada, de acordo com a norma de orientação clínica do IQS. ${ }^{3}$

\section{Material e Métodos}

Três anos após a abertura da consulta decidiu-se estudar os pacientes fumadores que tinham tido uma primeira consulta pelo menos há um ano. Obteve-se um grupo de 184 fumadores. Cada consulta foi registada em ficha clínica. Tendo por base os dados de preenchimento foi possivel obter as seguintes variáveis: sexo, idade, grupo profissional, grau de escolaridade, hábitos tabágicos, antecedentes pessoais e familiares, medicação crónica, nível de dependência da nicotina, razões para fumar, motivação para deixar de fumar, terapêutica farmacológica e taxa de abstinência tabágica. Os fumadores foram inquiridos sobre a existência de patologias crónicas. Nos antecedentes pessoais considerou-se como caso positivo, o fumador que apresentasse uma ou mais patologias crónicas do conjunto classificável.

Foi pedido ao fumador que graduasse as razões que o levam a fumar, assinalando com um círculo a sua resposta, de tal modo que 0 (zero) significava "nunca" e 10 (dez) significava "sempre". Para avaliação do nivel da dependência da nicotina utilizou-se o Teste de Fagerström. Este teste tem seis questões, cujas respostas permitem obter uma pontuação final cujo intervalo vai de 0 (zero) a 10 (dez) pontos, classificando a dependência em ligeira, moderada ou elevada.

A motivação do fumador para deixar de fumar foi avaliada por duas escalas, uma analógica com 10 (dez) pontos, em que 0 (zero) significava "sem nenhuma vontade» e 10 (dez) significava "francamente decidido a parar", outra numérica, o Teste de Richmond, com quatro questões e uma pontuação final de 0 a 10 , que avalia igualmente a motivação do fumador para parar de fumar e construir um plano concreto para o efeito. As duas escalas são complementares e a sua concordância é relevante.

À totalidade dos fumadores foi proposto um plano personalizado cognitivo-comportamental com o objectivo de cessarem o consumo de tabaco. A todos foi entregue um folheto informativo sobre tabaco/saúde/doença e o processo de cessação tabágica, com conselhos úteis e adaptado à fase de preparação/acção em que a maioria se encontrava. O médico de uma forma personalizada contratualizou com o fumador um conjunto de metas a cumprir e a avaliar em consultas futuras.

Mediante consulta médica presencial 
ou telefonema todos os fumadores inscritos na consulta há mais de um ano foram inquiridos quanto ao consumo de tabaco. Não foi realizada validação dos resultados por análise biológica.

Foi construída uma base de dados no programa Access. O estudo estatístico usou a aplicação informática SPSS. Para a avaliação da associação entre duas variáveis utilizou-se o teste de $\chi^{2}$. Para a comparação entre várias médias utilizou-se a análise de variância. Foi considerado um nivel de significância de 0,05.

\section{REsULTADOS}

Revisto o movimento de inscritos na consulta nos três anos considerados 2002, 2003 e 2004 conclui-se que o total de inscritos foi estável - 91, 94 e 85 utentes respectivamente. O total de consultas nos mesmos anos foi de 236 , 353 e 280, o que dá uma média de 3 consultas por inscrito. Os homens tiveram uma média de consultas de 3,3 ligeiramente superior à média das mulheres, de 2,8 com um desvio padrão de 2,3 e 1,8 respectivamente.

\section{CaracterizaCÃo dos Fumadores}

Apresentam-se algumas características sócio-demográficas dos fumadores (Quadro I).

Foram estudados 184 indivíduos, 92 (50\%) de cada sexo. A idade média dos fumadores estudados é de 45,7 anos, com um mínimo de 18 anos e um máximo de 82 anos. Distribuídos por grupos etários, o grupo dos 35-44 anos é o mais frequente com $32 \%$. É de referir o limitado número de jovens com menos de 25 anos (3,8\%) e o número de idosos com mais de 64 anos (9,3\%) que procuraram a consulta. A larga maioria dos fumadores exerce uma profissão (73,3\%). Os fumadores pertencem a

\begin{tabular}{|c|c|c|c|c|}
\hline \multicolumn{5}{|c|}{ QUADRO I } \\
\hline \multicolumn{5}{|c|}{$\begin{array}{l}\text { CARACTERÍSTICAS DOS FUMADORES POR SEXO, } \\
\text { IDADE, ESCOLARIDADE E PROFISSÃO }\end{array}$} \\
\hline & \multicolumn{4}{|c|}{ Sexo } \\
\hline & \multicolumn{2}{|c|}{ Feminino } & \multicolumn{2}{|c|}{ Masculino } \\
\hline & $n$ & $\%$ & $n$ & $\%$ \\
\hline \multicolumn{5}{|l|}{ Idade } \\
\hline $15-24$ & 5 & 2,7 & 2 & 1,1 \\
\hline $25-34$ & 18 & 9,8 & 9 & 4,9 \\
\hline $35-44$ & 26 & 14,1 & 33 & 17,9 \\
\hline $45-54$ & 24 & 13,0 & 23 & 12,5 \\
\hline $55-64$ & 13 & 7,1 & 14 & 7,6 \\
\hline $65+$ & 6 & 3,3 & 11 & 6,0 \\
\hline \multicolumn{5}{|l|}{ Escolaridade } \\
\hline Licenciatura & 29 & 15,8 & 18 & 9,8 \\
\hline Média & 17 & 9,2 & 11 & 6,0 \\
\hline Secundária & 37 & 20,1 & 43 & 23,4 \\
\hline Primária & 8 & 4,3 & 20 & 10,9 \\
\hline Analfabeto & 1 & 0,5 & 0 & 0,0 \\
\hline \multicolumn{5}{|l|}{ Profissão } \\
\hline Activa & 70 & 38 & 65 & 35,3 \\
\hline Desemprego & 10 & 5,4 & 9 & 4,9 \\
\hline Em formação & 5 & 2,7 & 1 & 0,5 \\
\hline Reforma & 5 & 2,7 & 17 & 9,2 \\
\hline Outro & 2 & 1,1 & 0 & 0,0 \\
\hline Total & 92 & 50,0 & 92 & 50,0 \\
\hline
\end{tabular}

uma população escolarizada em que, a maioria tem o grau de escolaridade secundária (43,5\%). É de realçar a percentagem de licenciados $(25,6 \%)$ e em particular entre os fumadores do sexo feminino $(15,8 \%)$.

\section{Caracterização do Hábito TabágICO}

A média da idade de consumo do primeiro cigarro foi de 15,6 anos (com um mínimo de 3 e um máximo de 38). No sexo masculino o início foi mais precoce (14,9 anos) do que no sexo feminino, sendo a diferença estatisticamente significativa ( $\mathrm{p}=0,021)$ (Quadro II).

O número médio de cigarros fumados por dia foi de 28,8 cigarros, com um mínimo de 8 e um máximo de 80 . A ca- 


\section{Artigos Breves}

\section{QUADRO II}

\begin{tabular}{|l}
\hline \multicolumn{4}{|c|}{ QUADRO II } \\
\begin{tabular}{|l|c|c|c|c|}
\hline \multicolumn{4}{|c|}{ IDADE DO PRIMEIRO CIGARRO POR SEXO } \\
\hline \multirow{2}{*}{$\begin{array}{l}\text { Idade do } \\
\text { primeiro } \\
\text { cigarro }\end{array}$} & Sexo & $\mathbf{n}$ & Média & $\mathbf{p}$ \\
\cline { 2 - 6 } & Feminino & 92 & 16,3 & \multirow{2}{*}{0,021} \\
\cline { 2 - 6 } & Masculino & 92 & 14,9 & \\
\cline { 2 - 6 } & Total & 184 & 15,6 & \\
\hline
\end{tabular}
\end{tabular}

\section{QUADRO III}

CARACTERIZAC̄̃O DOS HÁBITOS TABÁGICOS POR SEXO E GRUPO ETÁRIO

\begin{tabular}{|l|c|c|c|c|c|}
\hline \multirow{2}{*}{} & \multicolumn{5}{|c|}{$\mathbf{N}$ de cigarros/dia } \\
\cline { 2 - 6 } & $\leq 10$ & $11-20$ & $21-30$ & $\geq 31$ & \\
& $\mathbf{n}(\%)$ & $\mathbf{n}(\%)$ & $\mathbf{n}(\%)$ & $\mathbf{n}(\%)$ & $\mathbf{p}$ \\
\hline Sexo & & & & & \\
Feminino & $7(3,8)$ & $47(25,5)$ & $16(8,7)$ & $22(12,0)$ & 0,018 \\
Masculino & $4(2,2)$ & $29(15,8)$ & $21(11,4)$ & $38(20,7)$ & \\
\hline Total & $11(6,0)$ & $76(41,3)$ & $37(20,1)$ & $60(32,6)$ & \\
\hline Grupo etário & & & & & \\
$15-24$ & $2(1,1)$ & $2(1,1)$ & $1(0,5)$ & $2(1,1)$ & \\
$25-34$ & $2(1,1)$ & $11(6,0)$ & $5(2,7)$ & $9(4,9)$ & \\
$35-44$ & $1(0,5)$ & $27(14,7)$ & $11(6,0)$ & $20(10,9)$ & 0,29 \\
$45-54$ & $3(1,6)$ & $16(8,7)$ & $10(5,4)$ & $18(9,8)$ & \\
$55-64$ & $2(1,1)$ & $9(4,9)$ & $9(4,9)$ & $7(3,8)$ & \\
$65+$ & $1(0,5)$ & $11(6,0)$ & $1(0,5)$ & $4(2,2)$ & \\
\hline
\end{tabular}

racterização do hábito tabágico entre fumadores, definida como o número de cigarros fumados por dia, é apresentada segundo o sexo e o grupo etário (Quadro III). A maioria dos fumadores $(41,3 \%)$ fumava entre 11 e 20 cigarros, mas cerca de um terço $(32,6 \%)$ fumava mais de 31 cigarros. As mulheres fumavam menos quantidade de cigarros por dia (11 a 20) que os homens (31 ou mais), sendo a diferença significativa $(\mathrm{p}=0,018)$. A maioria dos fumadores do grupo etário dos 35-44 anos fumava 11 a 20 cigarros por dia $(14,7 \%)$ e 31 ou mais cigarros por dia $(10,9 \%)$.

\section{ANTECEDENTES PESSOAIS}

Entre os fumadores, 149 (81\%) apresentavam pelo menos uma patologia crónica diagnosticada. Acresce que 57 (31\%) referia um diagnóstico de patologia psiquiátrica. Esta é mais frequente no sexo feminino $(17,9 \%)$ e no grupo etário dos 35-44 anos (32,1\%). Em 110 casos (60\%) houve referência à toma regular de medicamentos.

\section{DEPENDÊNCIA DA NICOTINA}

Aplicado o Teste de Fagerström, a média dos totais obtida foi de 5,9 pontos.

Se agregarmos os resultados em três níveis de pontuação obtemos $17 \%$ dos fumadores com um nível ligeiro (pontuação menor que 4), 55\% com um nível moderado (pontuação entre 4 e 7) e 28\% dos fumadores com um nivel elevado (pontuação entre 8 e 10). Apresentam-se os resultados totais e por sexo dos 


\section{QUADRO IV}

\begin{tabular}{|c|c|c|c|c|}
\hline \multicolumn{5}{|c|}{$\begin{array}{l}\text { DEPENDÊNCIA DA NICOTINA ENTRE OS FUMADORES } \\
\text { POR SEXO, SEGUNDO O TESTE DE FAGERSTRÖM }\end{array}$} \\
\hline \multirow{4}{*}{$\begin{array}{l}\text { Pontuação } \\
\text { no Teste de } \\
\text { Fagerström }\end{array}$} & Sexo & n & Média & p \\
\hline & Feminino & 92 & 5,3 & \multirow{2}{*}{0,001} \\
\hline & Masculino & 92 & 6,4 & \\
\hline & Total & 184 & 5,9 & \\
\hline
\end{tabular}

fumadores. É de realçar que os fumadores do sexo masculino apresentam um nível superior de dependência $(6,4$ pontos) quando comparados com os do sexo feminino (5,3 pontos). Esta diferença é estatisticamente significativa $(\mathrm{p}=0,001)$ (Guadro IV e V).

\section{Razões PARA Fumar}

A principal razão apresentada para fumar foi "para combater o stresse", seguida "por prazer" e "por convivio social". A razão menos pontuada foi "para não engordar".

Estudadas as razões para fumar, por sexo dos fumadores, verificamos que no sexo feminino a primeira razão apontada é "para combater o stresse», enquanto que no sexo masculino foi "por prazer». São significativas as diferenças entre sexos quanto às razões "para combater o stresse" $(\mathrm{p}=0,002)$ e "para não engordar" $(\mathrm{p}=0,002)$, com predomínio de respostas afirmativas entre as mulheres (Quadro VI).

\section{Motivacão para DeIXAR de Fumar}

Os fumadores estudados apresentavam um nível de motivação para deixar de fumar de 8,3 pontos, segundo a escala analógica e um valor de 7,2 segundo o Teste de Richmond. Os resultados de ambas as escalas, segundo o sexo dos fumadores, não apresentam diferenças significativas.

\begin{tabular}{|} 
QUADRO V \\
\begin{tabular}{|c|c|c|}
\hline \multicolumn{3}{|c|}{ PONTUAÇ̃O NO TESTE DE FAGERSTRÖM } \\
ENTRE FUMADORES \\
\hline \hline Pontuação no Teste de Fagerström & $\mathbf{n}$ & $\%$ \\
\hline 0 & 2 & 1 \\
\hline 1 & 3 & 2 \\
\hline 2 & 11 & 6 \\
\hline 3 & 15 & 8 \\
\hline 4 & 20 & 11 \\
\hline 5 & 31 & 17 \\
\hline 6 & 28 & 15 \\
\hline 7 & 22 & 12 \\
\hline 8 & 25 & 14 \\
\hline 9 & 19 & 10 \\
\hline 10 & 8 & 4 \\
\hline
\end{tabular} \\
\hline
\end{tabular}

\section{Plano de Actuaç̃o}

O médico, tendo por base critérios previamente estabelecidos na equipa, referenciou 15 (8\%) dos fumadores à psicóloga e 28 (15\%) à nutricionista. Em 37 (20\%) fumadores foram propostas outras actuações, como referência a outras consultas de especialidade, prescrição de medicamentos, de actividade fisica, etc.

\section{TERAPÊUTICA FARMACOLÓGICA}

A 73 fumadores (40\%) foi prescrita te-

QUADRO VI

RAZÕES PARA FUMAR POR SEXO, SEGUNDO SETE ITEMS NUMA ESCALA DE 10 PONTOS

\begin{tabular}{|l|c|c|c|c|}
\hline \multirow{2}{*}{ Items } & \multirow{4}{|c|}{ Sexo } & \multirow{2}{*}{} \\
\cline { 3 - 4 } & Total & Feminino & Masculino & $\mathbf{p}$ \\
\hline l. Gesto automático & 5,8 & 5,9 & 5,7 & 0,57 \\
\hline 2. Convívio social & 6,2 & 6,3 & 6,0 & 0,65 \\
\hline 3. Prazer & 6,9 & 7,0 & 6,8 & 0,63 \\
\hline 4. Combate a0 stresse & 7,2 & 7,9 & 6,5 & 0,002 \\
\hline 5. Concentração & 4,3 & 4,5 & 4,2 & 0,53 \\
\hline 6. Levantar a moral & 3,8 & 4,2 & 3,4 & 0,08 \\
\hline 7. Medo de engordar & 1,5 & 2,1 & 0,9 & 0,002 \\
\hline
\end{tabular}


QUADRO VII

\begin{tabular}{|c|c|c|c|c|c|c|}
\hline \multicolumn{7}{|c|}{ QUADRO VII } \\
\hline \multicolumn{7}{|c|}{$\begin{array}{l}\text { AVALIAC̣̃̃O DA CESSAC̣̃O TABÁGICA UM ANO } \\
\text { APÓS A PRIMEIRA CONSULTA, POR SEXO }\end{array}$} \\
\hline & \multirow{2}{*}{\multicolumn{2}{|c|}{ Total }} & \multicolumn{4}{|c|}{ Sexo } \\
\hline & & & \multicolumn{2}{|c|}{ Feminino } & \multicolumn{2}{|c|}{ Masculino } \\
\hline & $n$ & $\%$ & $n$ & $\%$ & $n$ & $\%$ \\
\hline Fuma & 91 & 49 & 48 & 26 & 43 & 23 \\
\hline Não fuma & 44 & 24 & 16 & 9 & 28 & 15 \\
\hline Não responde & 49 & 27 & 28 & 15 & 21 & 11 \\
\hline
\end{tabular}

\section{QUADRO VIII}

AVALIACÃO DA CESSACÃO TABÁGICA UM ANO APÓS A PRIMEIRA CONSULTA, ENTRE OS FUMADORES COM E SEM PATOLOGIA PSIQUIÁTRICA

\begin{tabular}{|c|c|c|c|c|c|}
\hline & \multicolumn{2}{|c|}{$\begin{array}{c}\text { Sem } \\
\text { psicopatologia }\end{array}$} & \multicolumn{2}{|c|}{\begin{tabular}{|c} 
Com \\
psicopatologio
\end{tabular}} & \multirow[b]{2}{*}{$p$} \\
\hline & $n$ & $\%$ & $n$ & $\%$ & \\
\hline Fuma & 59 & 32 & 32 & 17 & \\
\hline Não fuma & 37 & 20 & 7 & 4 & 0,046 \\
\hline Não responde & 31 & 17 & 18 & 10 & \\
\hline
\end{tabular}

rapêutica farmacológica. Entre estes, a 50 ( $20 \%$ do total) foi prescrita terapêutica de substituição nicotínica (TSN) na apresentação de pastilhas mastigáveis e/ou sistemas transdérmicos e a 23 (13\%) foi prescrita a bupropiona. Em nenhum caso foi referido efeito secundário que impusesse a interrupção da medicação.

\section{Avalaç̃̃o da Consulta}

Doze meses após a data da primeira consulta 44 (24\%) estavam sem fumar, $91(49 \%)$ continuavam a fumar e em relação a 49 (27\%) não foi possível obter informação (Quadro VII).

No subgrupo de fumadores com patologia psiquiátrica, constituído por 57 fumadores $(31 \%)$, os resultados foram que $32(17 \%)$ se mantiveram a fumar, em $18(10 \%)$ não foi possível obter informação e 7 (4\%) deixaram de fumar.
A comparação entre os dois grupos confirma que os fumadores com patologia psiquiátrica tiveram menor êxito em deixar de fumar $(\mathrm{p}=0,046)$ (Quadro VIII).

\section{DIscussão}

Foram estudados 184 fumadores inscritos na consulta de cessação tabágica do Centro de Saúde de Alvalade. Os fumadores correspondem à totalidade dos inscritos que realizaram a primeira consulta há pelo menos 12 meses.

É relevante o elevado número de fumadores do sexo feminino que recorreram à consulta. Embora em Portugal exista uma relação de 4 homens para 1 mulher entre os fumadores, ${ }^{4}$ o resultado encontrado é concordante com os dados da literatura que nos apontam as mulheres como as que mais cuidados de saúde procuram, quer preventivos quer curativos, independentemente da patologia considerada ${ }^{5}$ e também quando se trata do tabagismo. ${ }^{6}$

Quanto à profissão, salienta-se a percentagem de desempregados encontrada $(10,3 \%)$, pois esta situação é conhecida como um factor de insucesso na cessação tabágica, ${ }^{7}$ entre outras razões pelo elevado número de cigarros que fumam e a falta de recursos para custear os medicamentos. ${ }^{8}$

Sabemos que em Portugal a prevalência de mulheres fumadoras tende a ser superior nas mais escolarizadas, surgindo o consumo de tabaco associado a um estatuto social superior. ${ }^{4}$ Os resultados obtidos confirmam que, em geral, são os indivíduos mais escolarizados que primeiro solicitam apoio para deixar de fumar o que é concordante com o desenvolvimento da epidemia tabágica em Portugal. Actualmente, esta epidemia encontra-se entre as fases 2 e 3 , com $35 \%$ de homens e $18 \%$ das mulheres fumadoras e com a mortalidade por cancro do pulmão ainda muito baixa no sexo feminino, aproximando-se 
de outros países do sul da Europa que estão já na terceira fase. ${ }^{9}$

A idade de consumo do primeiro cigarro é semelhante aos valores publicados para Portugal. ${ }^{4}$ No entanto, o número de cigarros fumados por dia é superior ao número encontrado no Inquérito Nacional de Saúde de 1998/99 (19 $\mathrm{cig} / \mathrm{dia})^{4}$ e ao número médio europeu (23 cig/dia). ${ }^{8}$

Não é fácil comparar a taxa de abstinência obtida com a de outros estudos nacionais pois os poucos estudos publicados têm modelos de prática diferentes. Quanto às taxas encontradas em estudos internacionais, estas não diferem muito. West e colaboradores, em 2000, num artigo de revisão, publicaram taxas de abstinência de longo prazo de $18 \%$ para consultas de apoio intensivo com prescrição de substitutos de nicotina. ${ }^{10}$ Grandes e colaboradores, em 2003, num trabalho realizado em centro de saúde e também em consultas de tipo semelhante, obtiveram taxas de $22 \% .{ }^{11}$ Steinberg e colaboradores, em 2006, publicaram um extenso estudo, o "Lung Health Study" em que com a combinação de consultas de apoio particularmente intensivo e a prescrição de substitutos de nicotina conseguiram taxas de abstinência a um ano de $35 \%,{ }^{13}$ uma das mais altas taxas descritas na literatura.

\section{REFERÊNCIAS BIBIUUOGRÁFICAS}

1. World Health Organization Europe. Which are the most effective and cost-effective interventions for tobacco control? WHO Regional Office for Europe's Health Evidence Network (HEN). Geneva: WHO; 2003.

2. Rebelo L. Consulta de cessação tabágica: uma proposta para a Medicina Geral e Familiar. Rev Port Clin Geral 2004 Jan-Fev; 20 (1): 87-98.

3. Soares I, Carneiro AV. Norma de orientação clínica prática para o tratamento do uso e dependência do tabaco. Lisboa: Instituto da Qualidade em Saúde; 2002.

4. Ministério da Saúde. Instituto Nacional de Saúde Dr. Ricardo Jorge. Inquérito Nacional de Saúde 1998/99. Lisboa: INSA; 2000.

5. Cabral MV, Silva PA, Mendes H. Saúde e doença em Portugal. Lisboa: Instituto de Ciências sociais - Universidade de Lisboa; 2002.

6. Boyle P, Gandini S, Robertson C, Zatonski W, Fagerstrom K, Slama K, et al. Characteristics of smokers' attitudes towards stopping. Survey of 10,295 smokers in representative samples from 17 European countries. Eur J Pub Health 2000 ; 10 (3 Suppl): 5-14.

7. Fiore MC, Bailey WC, Cohen SJ. Treating tobacco use and dependence: Clinical Practice Guideline. Rockville: US Department of Health and Human Services; 2000.

8. European Opinion Research Group. Special Eurobarometer: Europeans on smoking and the environment: actions and attitudes. Brussels: European Commission; 2003.

9. Santos AC, Barros H. Smoking patterns in a community sample of Portuguese adults, 1999-2000. Prev Med 2004 Jan; 38 (1): 114-9.

10. West R, McNeill A, Raw M. Smoking cessation guidelines for health professionals: an update. Thorax 2000 Dec; 55 (12): 987-99.

11. Grandes G, Cortada JM, Arrazola A, Laka JP. Predictors of long-term outcome of a smoking cessation programme in primary care. Br J Gen Pract 2003 Feb; 53 (487): 101-7.

12. Steinberg MB, Foulds J, Richardson DL, Burke MV, Shah P. Pharmacotherapy and smoking cessation at a tobacco dependence clinic. Prev Med 2006 Feb; 42 (2): 114-9.

13. Anthonisen NR, Connett JE, Kiley JP, Altose MD, Beiley WC, Buist AS, et al. Effects of smoking intervention and use of an inhaled anticholinergic bronchodilator in the rate of decline of FEV 1. The Lung Health Study. JAMA 1994 Nov 16; 272 (19): 1497-505.

\section{Endereço para correspondência:}

Luís Rebelo

Rua Maria Veleda Torre $2-5^{\circ} \mathrm{A}$

1500-441 Lisboa

E-mail: 1rebelo@sapo.pt

Recebido em 16/07/2007

Aceite para publicação em 11/02/2008 


\section{Artigos Breves}

\section{SMOKING CESSATION SERVICE IN ALVALADE HEALTH CENTER (LISBON). THE FIRST 184 SMOKING PATIENTS. EVALUATION OF RESULTS.}

\section{ABSTRACT}

Objectives: To characterize the Smoking Cessation Service of the Alvalade Health Center and to evaluate the abstinence rate among smoking patients.

Methodology: Descriptive and cross-sectional study. Data was collected from the clinical registries of all the 184 smoking patients who had completed, by the time of the study, at least one year after the first appointment. Fagerstrom test was used to evaluate physical dependence on nicotine and Richmond test was used to determine motivation to stop smoking.

Results: The studied smokers belong to both genders, have a medium age of 45 years, have a professional activity and a secondary level of education. The majority smoked the first cigarette at the age of 15 and smoked an average of 28 cigareftes per day. Women smoked less than men, between 11 and 20 cigarettes versus 30 or more $(p=0.018)$. The majority showed a moderate dependence on nicotine (a score of 5.9). Men scored higher than women, 6.4 versus 5.3 ( $p=0.001)$. Women smoked as a coping mechanism "to manage stress" and men smoked "for pleasure". When compared to men, "managing stress" ( $p=0.002)$ and "weight control" $(p=0.002)$ are smoking reasons referred by women. Abstinence rate, evaluated 12 months after the first appointment, was $24 \%$. Smokers with psychiatric pathology had more difficulty quitting smoking $(p=0.046)$.

Conclusions: The studied smokers belong to both genders, have a medium age of 45 years, a medium level of education, and began to smoke by the age of 15 . They smoke 28 cigarettes per day, show a moderate dependence on nicotine and are motivated to stop smoking. Twelve months after the first appointment, $24 \%$ of them were abstinent.

Keywords: Tobacco; Service; Smoking Cessation. 